\title{
Review of: "Smoking, vaping and hospitalization for COVID-19"
}

John Stapleton

The authors should be commended for continuing to refine their work with addition data, an improved analytical strategy, and a more scholarly discussion. Their new focus on nicotine (and possibly its metabolites?) also seems appropriate.

In response to an earlier version (v10), I expressed concern about the analytical approach (now well addressed), and the apparent paradox of roughly equal numbers of hospitalised males and females observed, given that in the population approximately half of males smoke, against a near neg ligible prevalence in women. On this latter point, if smoking (or one of its constituents - such as nicotine) are protective, then we would expect to see fewer males than those observed in Chinese admissions. This issue was addressed by Prof. Hajek in his comments, although it does not seem to have been incorporated by the authors in more recent versions. How can the hospitalised sex prevalence ratio be viably adjusted to arrive at the data presented? If we are prepared to work in rough rates and numbers, the below reasoning might be credible.

The authors review of Chinese data suggests only $1 / 4$ as many smokers as nonsmokers are hospitalised as a result of CV symptoms. Alone, this would result in far fewer Chinese males being hospitalised than the 57\% observed in the review. This difference (in its simplest form) can only be resolved if an independent (of smoking) and detrimental male effect that can be identified. Estimates of this detrimental "male" symptomology, sufficient for hospitalisation, vary from around the world. Professor Hajek cited a 38\% vs $62 \%$ female : male ratio in Chinese data. Of course, with a 20 or 25 -fold sex difference in smoking rates in this population, "independence" of sex and smoking is a hazardous assumption. As he suggested, we need to look at populations where smoking is near equal in males and females to obtain a cleaner estimate of any male : female effect. The UK ONS have recently published data suggesting that among CV-related deaths the ratio is nearer 2:1, which would certainly be sufficient to explain the inconsistencies in the authors' data if we can assume that the sex ratio of hospitalised individuals is the same as in those that later die. There are likely more data on this aspect which the authors might access, in addition to the Italian data ( $68 \%$ vs $32 \%$, male : female deaths ) that they 
cite in reply to other comments. However, if we proceed with this reasoning, by assuming that about 2/3 as many "cases" in women as men are sufficiently ill to be hospitalised (independently of smoking), and that 1/4 as many smokers as non-smokers are hospitalised (independent of sex, authors' data), then we can reconcile the rates presented in this review, with about $57 \%$ of those hospitalised being male. On the basis of these crude calculations, I would urge the authors to look again at this issue and include it in their discussion analysis.

The issue raised by myself and others regarding the definition of "current smoker" in these data remains a major concern, even if the sex-ratio issue can be adequately reconciled. The authors have offered some reassurance on this, but need to go further by obtaining additional information - unbiased and honest - from the study authors, as seems to have been achieved by Prof. Larkin. If they can, they might close the door on the more obvious concerns and achieve a report that would be truly worthy of closer investigation by those with the resources to do so. 